\title{
Integrative Analysis of the lncRNA and mRNA Transcriptome Revealed Genes and Pathways Potentially Involved in the Anther Abortion of Cotton (Gossypium hirsutum L.)
}

\author{
Yuqing Li ${ }^{1,2}$, Tengfei Qin ${ }^{2}$, Na Dong ${ }^{2}$, Chunyan Wei ${ }^{2}$, Yaxin Zhang ${ }^{2}$, Runrun Sun ${ }^{2}$, Tao Dong ${ }^{2}$, \\ Quanjia Chen ${ }^{1}$, Ruiyang Zhou ${ }^{3, *}$ and Qinglian Wang ${ }^{2, *}$ \\ 1 Cotton Engineering Research Center of Ministry of Education, College of Agriculture, \\ Xinjiang Agricultural University, Xinjiang Urumqi 830000, China; lyq120327@163.com (Y.L.); \\ chqjia@126.com (Q.C.) \\ 2 Collaborative Innovation Center of Modern Biological Breeding of Henan Province, Henan Key Laboratory \\ Molecular Ecology and Germplasm Innovation of Cotton and Wheat, School of Life Science and Technology, \\ Henan Institute of Science and Technology, Henan Xinxiang 453003, China; qintengfeisam@163.com (T.Q.); \\ dongna1229@163.com (N.D.); weichunyan688@163.com (C.W.); 18339511268@163.com (Y.Z.); \\ sunrunrun123@163.com (R.S.); dongtao985@163.com (T.D.) \\ 3 Key Laboratory of Plant Genetics and Breeding, College of Agriculture, Guangxi University, \\ Nanning 530005, China \\ * $\quad$ Correspondence: ruiyangzh@aliyun.com (R.Z.); wangq1985@163.com (Q.W.); Tel.: +86-137-0373-8603 (Q.W.)
}

Received: 28 September 2019; Accepted: 18 November 2019; Published: 20 November 2019

\begin{abstract}
Cotton plays an important role in the economy of many countries. Many studies have revealed that numerous genes and various metabolic pathways are involved in anther development. In this research, we studied the differently expressed mRNA and lncRNA during the anther development of cotton between the cytoplasmic male sterility (CMS) line, C2P5A, and the maintainer line, $\mathrm{C} 2 \mathrm{P} 5 \mathrm{~B}$, using RNA-seq analysis. We identified 17,897 known differentially expressed (DE) mRNAs, and $865 \mathrm{DE}$ long noncoding RNAs (lncRNAs) that corresponded to 1172 cis-target genes at three stages of anther development using gene ontology (GO), Kyoto Encyclopedia of Genes and Genomes (KEGG) enrichment of DE mRNAs; and cis-target genes of DE lncRNAs probably involved in the degradation of tapetum cells, microspore development, pollen development, and in the differentiation, proliferation, and apoptosis of the anther cell wall in cotton. Of these DE genes, LTCONS_00105434, LTCONS_00004262, LTCONS_00126105, LTCONS_00085561, and LTCONS_00085561, correspond to cis-target genes Ghir_A09G011050.1, Ghir_A01G005150.1, Ghir_D05G003710.2, Ghir_A03G016640.1, and Ghir_A12G005100.1, respectively. They participate in oxidative phosphorylation, flavonoid biosynthesis, pentose and glucuronate interconversions, fatty acid biosynthesis, and MAPK signaling pathway in plants, respectively. In summary, the transcriptomic data indicated that DE lncRNAs and DE mRNAs were related to the anther development of cotton at the pollen mother cell stage, tetrad stage, and microspore stage, and abnormal expression could lead to anther abortion, resulting in male sterility of cotton.
\end{abstract}

Keywords: cotton; cytoplasmic male sterility; lncRNA; transcriptome; cis-target gene

\section{Introduction}

In recent years, with the development of sequencing technology, numerous genes and gene families have been identified. In addition, some transcriptomes, which were previously regarded 
as being "dark" or "junk" having either no or only a weak protein-coding ability, also have been identified [1-3]. However, multiple functions have recently been discovered for noncoding regions.

Long noncoding RNA (lncRNA) is considered a part of the "dark" or "junk" transcriptomes. In previous studies, IncRNA was underestimated and even considered to be transcriptional noise because of its low expression level and weak sequence conservation, when compared to mRNA [4]. Generally, lncRNAs are defined as being larger than 200 nucleotides in length, and play vital regulatory roles in biological processes in plants and animals [5-7], such as plant growth and development, epigenetics, and stress response [5,7]. They have important regulatory functions in organisms, and are strictly regulated at the transcriptional and post-transcriptional levels [8]. Studies have shown that lncRNAs have high stage- and tissue-specific expression patterns $[9,10]$.

lncRNAs are categorized into three categories-long intergenic RNAs (lincRNAs), intronic ncRNAs (incRNAs), and natural antisense transcription (NATs)—based on the origin of genome $[4,11]$ and into four categories, such as lincRNAs, incRNAs, antisense ncRNAs (ancRNAs), and sense ncRNAs (slncRNAs) based on the genomic location of IncRNAs [12].

In previous studies, IncRNAs were shown to play pivotal roles in the stress responses of plant development. In Chinese cabbage, IncRNA regulates heat stress, and 4594 putative lncRNAs were identified by strand-specific RNA-sequencing [13]. In wheat, lncRNAs were responsive to powdery mildew infection and heat stress [14]. In ELF18-INDUCED LONG-NONCODING RNA1 (ELENA1) mutants of a factor enhancing resistance against Pseudomonas in Arabidopsis, long noncoding RNAs were implicated in the transcriptional regulation of plant innate immunity [15]. LncRNA16397 is resistant to Phytophthora infestans by co-expressing glutaredoxin in tomato [16]. LncRNAs affect the root development response to cadmium stress at an early stage of rice [17]. Phosphate deficiency-induced lncRNAs (PDILs) involved in Pi-deficiency signaling and Pi transport have shown that lncRNAs regulate the responses of plants to phosphate starvation [18,19]. LncRNAs are significantly altered under nitrogen deficiency in Populus [20].

Some fertility-related lncRNAs have been identified in plants in recent years. In rice, we obtained 2224 reliably expressed lncRNAs, including 1624 lincRNAs and 600 lncNATs involved in rice reproduction [10]; photoperiodic-sensitive male sterility (PSMS) is regulated by lncRNA long-day-specific male-fertility-associated RNA (LDMAR) [21], as well as lncRNAs MS1T transgenic plants associated with male sterility under long-day conditions [22]. In diploid strawberry, 5884 lncRNAs were identified from the strawberry RNA-seq data during flower and fruit development [23]. In trifoliate orange, genome-wide screening showed 6584 potential lncRNAs associated with flowering development [24]. In tomato, 79,322 putative lncRNAs were identified, consisting of 70,635 lincRNAs, 8085 ancRNAs, and 602 slncRNAs related to regulation during fruit ripening [12].

The Gossypium raimondii (Dt), Gossypium arboreum (At), and Gossypium hirsutum (AtDt) genomes are now sequenced [25-27], which has promoted a huge leap forward in cotton genomics [28]. With the development of modern technology, the de novo assembly of Gossypium hirsutum and Gossypium barbadense genomes in high quality has improved contiguity and completeness in the available genome information [29,30]. In cotton, IncRNAs participate in resistance to Verticillium dahliae [31], drought stress [32], salt stress [33], and other abiotic stresses [34]. LncRNAs also participate in regulating fiber development [5,35] and in lint initiation [36]. However, little is known about the mechanisms of lncRNA during the cytoplasmic male sterility (CMS) in cotton; therefore, it is necessary to identify novel lncRNA and analyze the lncRNA in the development of cotton anther.

In this research, the expression of mRNAs and lncRNAs during different stages of anther development in cotton CMS line C2P5A and maintainer line C2P5B was characterized using RNA-sequencing technology. Our results demonstrate the molecular mechanisms involving lncRNAs and mRNAs during the anther development of cotton between the CMS line and the maintainer line and thus provides new perspectives for the utilization of heterosis. 


\section{Materials and Methods}

\subsection{Plant Materials}

The CMS line C2P5A and maintainer line C2P5B of cotton (Gossypium hirsutum L.) are near-isogenic lines. Plants were cultivated in the experimental field, in Henan, China, under normal management conditions. Both lines were identified with 1.5\% (w/v) acetocarmine staining and observed using scanning electron microscopy (EPSON EXPRESSION 12000XL, Nagoya, Japan).

Three different stages of anther development-the pollen mother cell stage (Pms; 3-4 mm), tetrad stage (Tds; 4.1-5.0 mm), and mononuclear stage (Ms; 5.1-6.0 mm) - and flower buds with a certain length (length from nectary to bud apex; $\mathrm{mm}$ ) were selected for study. For pure anthers, we first peeled off the sepals and petals, discarded the pistil, and the resulting anthers were collected and placed in a centrifugal tube, which was frozen in liquid nitrogen and stored at $-80^{\circ} \mathrm{C}$ until use.

\subsection{Library Construction and RNA-Sequencing (RNA-Seq)}

Total RNA was extracted from the harvested anthers using an RNAprep Pure Plant Kit (Tiangen, Beijing, China) using three biological replicates per sample. The RNA concentration was detected by NanoDrop (Waltham, MA, USA), and quality was tested using an Agilent 2100 instrument (Santa Clara, CA, USA). cDNA library construction and sequencing were performed by Beijing Genomics Institute (Shenzhen, China) using the Illumina HiSeqTM 2000 system (Illumine, San Diego, CA, USA).

\subsection{Transcript Assembly, Alignment, and Identification of Genes}

In this study, raw reads were filtered to remove the adapter, low-quality reads, and contaminating sequences. Clean reads were then aligned to the reference genome (http://cotton.hzau.edu.cn/EN/ download.php) by HISAT [29,37] and Bowtie2 [38], and the transcript was assembled by String Tie [39]. For mRNA identification, uniquely mapped and properly paired reads were used in the transcript construction with Cufflinks, and the constructed transcripts were compared with the cotton (Gossypium hirsutum) gene annotation using Cuffcompare [40].

These transcripts were compared with known mRNAs and lncRNAs in order to obtain information about their location relationships using Cufflinks, and were then merged into the transcript assembly result using Cuffmerge [40]. FPKM (Fragments PerKilobase Million) was used to calculate gene expression levels by RSEM [41].

For lncRNA identification, we assembled a single transcript meeting the following requirements: sequence length $>200 \mathrm{bp}, \mathrm{FPKM}>0.5$, and coverage $>1$. The newly assembled transcript was compared to the cotton (Gossypium hirsutum) reference genome annotations so as to remove annotated transcripts (mRNA) and transcripts overlapping with other noncoding RNA species (e.g., tRNA, rRNA, snRNA, snoRNA, and miRNA) using Cuffcompare [42]. For the prediction of the transcript-coding ability, we used the Coding Potential Calculator (CPC) [43], txCdsPredict, and Coding-Non-Coding Index (CNCI) [44] - three predictive software-and a database Pfam [38]. The transcripts with a significant coding potential (CPC score $>0$, CNCI score $>0$, or $\mathrm{txCdsPredict}>500$ ) were discarded.

\subsection{Differential $m R N A$ and $\operatorname{lnc} R N A$ Expression Analyses}

The transcripts were aligned to the reference sequence with Bowtie2 [38], and RSEM used to calculate the expression levels of the genes and transcripts [41]. The differentially expressed genes (DEGs) were analyzed with DEGseq software [45]. Transcripts (mRNA or lncRNA) were normalized according to the $\mid \log _{2}$ Ratio $\mid \geq 2$, read number $>5, p \leq 0.001$, and FDR $<0.001$. GO annotation and KEGG pathway enrichment analysis were performed for the mRNA DEGs. 


\subsection{IncRNA-mRNA Pathway Network Construction}

LncRNAs can cis-regulate mRNAs, and it is believed that the function of lncRNA is related to its neighboring protein-coding genes [46]. We performed a location-expression analysis between mRNAs and lncRNAs in the $20 \mathrm{kbp}$ of sequence representing the region closest to the identified lncRNAs. We computed Pearson's correlation and Spearman's correlation coefficients between each pair of lncRNA-mRNA. The lncRNA-mRNA with the most significant correlations with coefficients of $>0.6$ were considered potential cis-target genes for those lncRNAs. The potential cis-target genes of the lncRNAs were subjected to enrichment analysis with GO annotation and KEGG pathway. DE IncRNAs' potential cis-target genes, terms, and pathways were visualized using Cytoscape v3.6 (http://www.cytoscape.org) [47].

\subsection{Gene Expression Confirmed by Real-Time Quantification PCR (RT-qPCR)}

RNA reverse transcription was conducted using TransScript ${ }^{\circledR}$ One-Step gDNA Removal and cDNA Synthesis SuperMix (TransGen, Beijing, China). The random selection of several genes related to sterility by RT-qPCR was used to verify the RNA-sequencing (RNA-seq) data. Primer Premier 6.0 software was used to design specific primers (http://www.premierbiosoft.com/crm/jsp/com/pbi/crm/ clientside/ProductList.jsp), which were synthesized by Sangon Biotech (Shanghai, China). The RT-qPCR reactions were performed with a qPCR SuperMix Kit (TransGen, Beijing, China). Each reaction was performed in three biological and three technical replicates on a QuantStudio 6 Flex instrument (Applied Biosystems, Foster City, CA, USA). RT-qPCR was performed according to the protocol of the TransStart ${ }^{\circledR}$ Top Green qPCR SuperMix in two steps (TransGen, Beijing, China). The expression levels were quantified relative to that of the housekeeping gene GhACT4 (GenBank accession no. AAP73451.1). The relative expression of each gene in every sample was calculated using the cycle threshold (Ct) $2^{-\Delta \Delta \mathrm{Ct}}$ method [48].

\section{Results}

\subsection{Identification and Characterization of $\ln c R N A$ and $m R N A$}

The RNA Seq data were uploaded to NCBI's SRA database (project code: PRJNA579288). A total of 1,394,119,526 clean reads were obtained by sequencing all 18 libraries. Each library produced an average of 11.62 million data. The clean reads were then matched to the cotton reference genome by HISAT. The average genome alignment rate was $76.98 \%$ (Table 1 ). A total of 178,166 transcripts were detected in 18 anther tissues of cotton, and 28,047 novel lncRNAs were identified using the following four programs: CPC, txCdsPredict, CNCI, and Pfam (Figure 1A); and 46,245 novel mRNAs and 103,874 known transcripts were identified. Many lncRNAs transcripts were mainly composed of 1-7 exons, whereas mRNAs had a wide range of exons, 1-10 (Figure 1B). The transcript lengths distribution of the lncRNAs was shorter than those of the mRNAs (Figure 1C). 
Table 1. Summary of tag numbers.

\begin{tabular}{lcccccccc}
\hline Sample & $\begin{array}{c}\text { Raw } \\
\text { Data }\end{array}$ & $\begin{array}{c}\text { Clean } \\
\text { Data }\end{array}$ & GC (\%) & Q30 (\%) & $\begin{array}{c}\text { Clean } \\
\text { Reads } \\
\text { Rate (\%) }\end{array}$ & $\begin{array}{c}\text { novel_lnc } \\
\text { RNA.isofo } \\
\text { rms }\end{array}$ & $\begin{array}{c}\text { novel_m } \\
\text { RNA.isofo } \\
\text { rms }\end{array}$ & $\begin{array}{c}\text { known_m } \\
\text { RNA.isofo } \\
\text { rms }\end{array}$ \\
\hline MsPms1 & 96339898 & 84112026 & 44.33 & 97.65 & 87.308 & 26669 & 22642 & 77144 \\
MsPms2 & 96339060 & 84728466 & 44.43 & 97.84 & 87.948 & 26079 & 22080 & 74534 \\
MsPms3 & 96340692 & 84822750 & 44.52 & 97.81 & 88.045 & 26723 & 22570 & 76486 \\
MsTds1 & 66418638 & 60016542 & 44.22 & 98.33 & 90.361 & 25569 & 22130 & 74937 \\
MsTds2 & 96341884 & 84562392 & 43.57 & 97.68 & 87.773 & 27125 & 23083 & 78797 \\
MsTds3 & 96340878 & 84830410 & 44 & 97.77 & 88.052 & 27515 & 22581 & 76691 \\
MsMs1 & 70226770 & 62112146 & 45.21 & 98.35 & 88.445 & 24684 & 21119 & 72145 \\
MsMs2 & 96340984 & 84269862 & 44.5 & 97.81 & 87.47 & 27175 & 22017 & 75181 \\
MsMs3 & 75363530 & 68729552 & 44.43 & 98.45 & 91.197 & 26502 & 22011 & 75483 \\
MPms1 & 78357630 & 71140510 & 43.98 & 98.42 & 90.79 & 26798 & 22389 & 74464 \\
MPms2 & 93592588 & 83300884 & 43.81 & 97.97 & 89.004 & 27279 & 22445 & 74560 \\
MPms3 & 94122576 & 81326894 & 44.24 & 97.83 & 86.405 & 27314 & 22397 & 74869 \\
MTds1 & 93071774 & 84922682 & 43.79 & 98.38 & 91.244 & 27246 & 22535 & 74405 \\
MTds2 & 79502370 & 72818484 & 43.85 & 98.23 & 91.593 & 26317 & 22163 & 73329 \\
MTds3 & 71210292 & 64700084 & 43.83 & 98.33 & 90.858 & 26046 & 21985 & 72553 \\
MMs1 & 96340778 & 84992800 & 44.36 & 98.03 & 88.221 & 26255 & 22543 & 75378 \\
MMs2 & 96340324 & 84734142 & 43.65 & 97.83 & 87.953 & 26319 & 22745 & 75915 \\
MMs3 & 74569750 & 67998900 & 43.6 & 98.23 & 91.188 & 25285 & 22181 & 73375 \\
\hline
\end{tabular}

MS, Male sterile line C2P5A; M, Maintainer line C2P5B; 1, 2, 3 represent the biological duplications; Pms, pollen mother cell stage; Tds, tetrad stage; Ms, mononuclear stage.
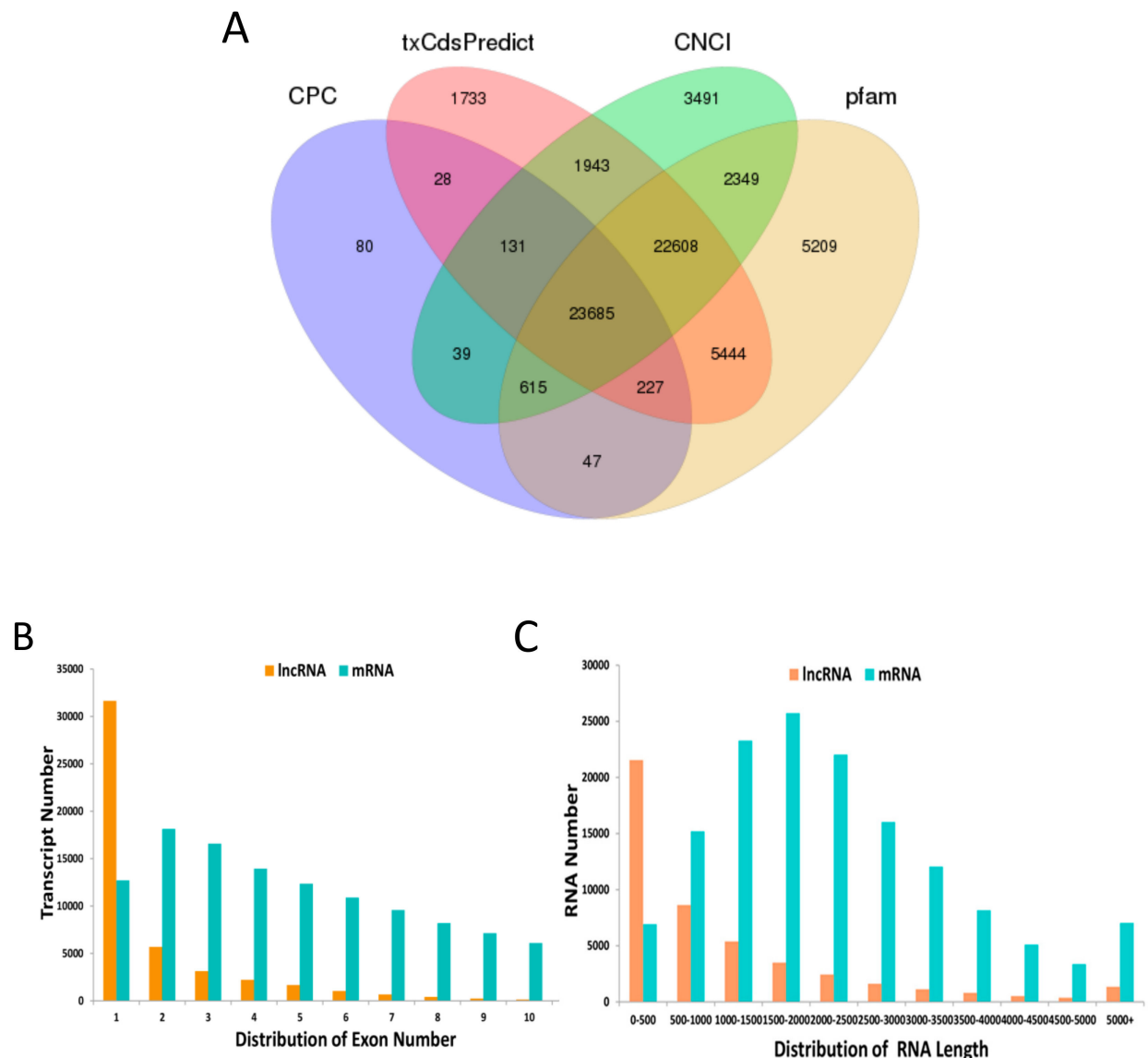

Figure 1. Long noncoding RNA (lncRNA) and mRNA characterization. (A) Venn diagram for screening results of the lncRNA by four software (Coding Potential Calculator (CPC), txCdsPredict, Coding-Non-Coding Index (CNCI), and Pfam). (B) The distribution of the lncRNA and mRNA exon number. (C) The distribution of lncRNA and mRNA length. 


\subsection{Differentially Expressed (DE) $m R N A$ s and $\ln c R N A s$}

A total of 6720, 7737, 9090 known mRNAs (Figure 2A, Table S1a-c) and 1689, 1657, and 2012 lncRNAs (known lncRNA 0, all novel lncRNAs; Figure 2B, Supplementary Table S2a-c) were DEGs between the CMS line and the maintainer line in the Pms, Tds, and Ms of anther development in cotton, respectively. Also, 1082 known mRNAs of DEGs and 189 lncRNAs were shared among the three stages (Tables S1d and S2d).
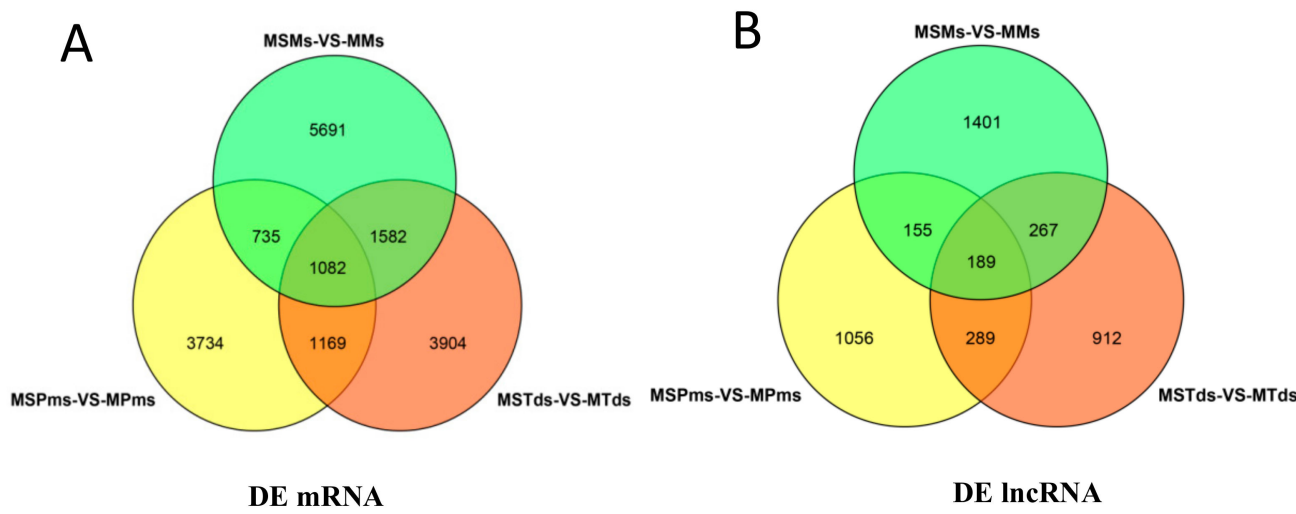

Figure 2. Venn diagram showing known mRNAs corresponding to differentially expressed genes (DEGs), and novel lncRNAs differentially expressed at three stages of anther development, between the cytoplasmic male sterility line C2P5A and the maintainer line C2P5B. (A) Venn diagram of known mRNAs of DEGs. (B) Venn diagram of novel lncRNA DEGs. MS-Male sterility line; M-Maintainer line; Pms—-pollen mother cell stage; Tds—-tetrad stage; Ms—mononuclear stage.

\subsection{DE mRNAs Enrichment Analyses}

A total of 27 GO terms and 22 KEGG enriched pathways were significantly enriched for DE mRNAs at the Pms stage between C2P5A and C2P5B (Table S3a,b, respectively). The top 20 terms were mostly enriched for malate synthase activity, catalytic activity, and carbohydrate metabolic process (Figure 3A and Table S3g). Moreover, the top 20 pathways were primarily enriched for several processes, such as flavonoid biosynthesis, plant hormone signal transduction, and fatty acid metabolism (Figure 3D and Table S3h).

A total of 32 GO terms and 22 KEGG-enriched pathways were significantly enriched for the DE mRNAs at the Tds stage between C2P5A and C2P5B (Table S3c,d, respectively). The top 20 terms of the GO were mostly enriched for oxidoreductase activity, malate synthase activity, and carbohydrate metabolic process (Figure 3B and Table S3g). Furthermore, the top 20 pathways of the KEGG were mainly enriched in starch and sucrose metabolism, plant hormone signal transduction, and flavonoid biosynthesis (Figure 3E and Table S3h).

At the Ms stage of anther development, between C2P5A and C2P5B, the DE mRNAs were significantly enriched for 66 terms (Table S3e), and the top 20 terms were mostly enriched in the carbohydrate metabolic process, oxidoreductase activity, and glucan metabolic process (Figure $3 \mathrm{C}$ and Table S3g). This was significantly enriched for the 20 KEGG pathway (Table S3f), and the top 20 terms mostly participated in plant hormone signal transduction, MAPK signaling pathway to the plant, flavonoid biosynthesis, and starch and sucrose metabolism (Figure 3F and Table S3h). 


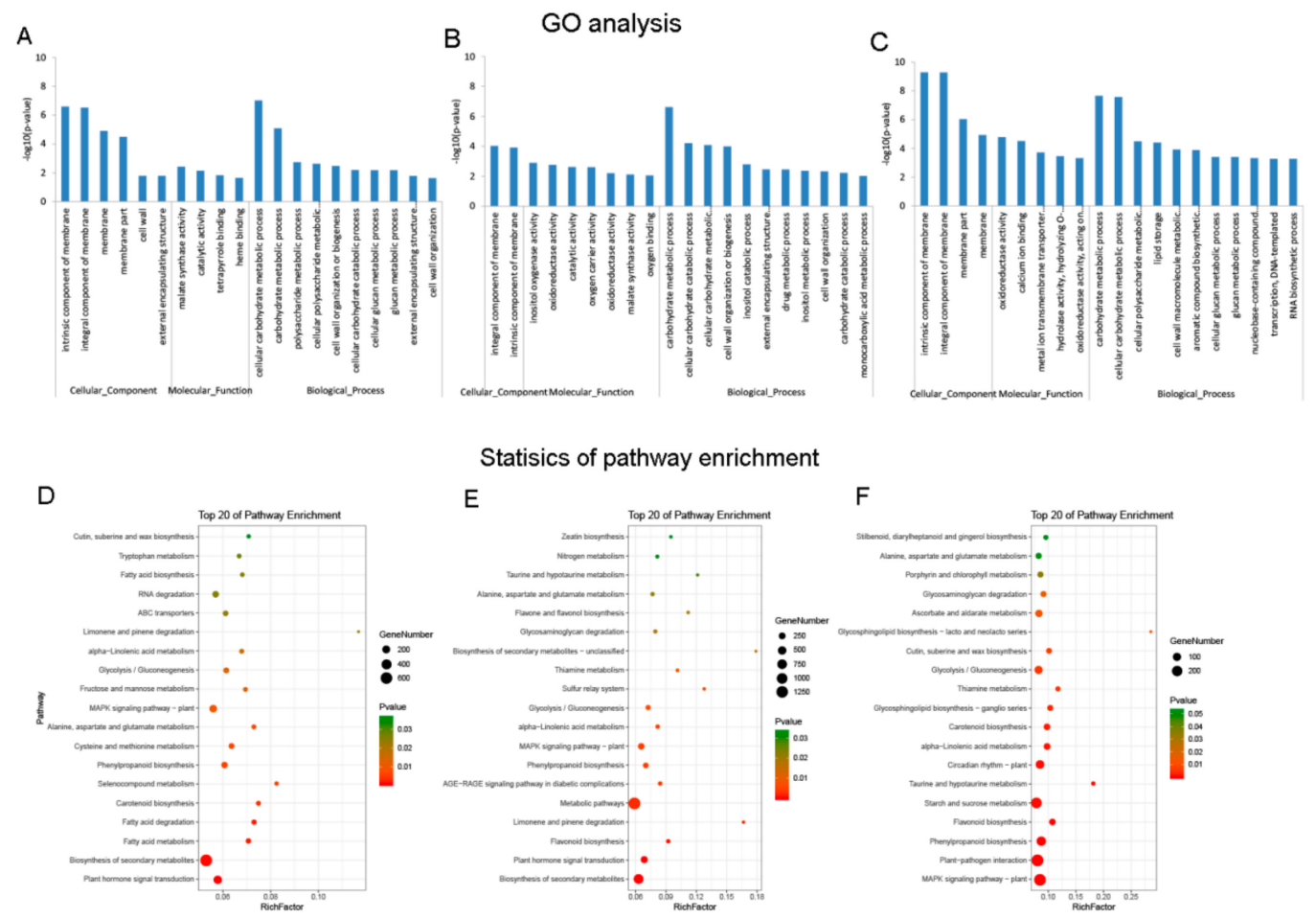

Figure 3. Differential expression of mRNAs at three stages of anther development, between the cytoplasmic male sterility line C2P5A and the maintainer line C2P5B. Gene ontology (GO) analysis of DEGs in (A) MSPms-VS-MPms, (B) MSTds-VS-MTds, and (C) MSMs-VS-MMs. Statistical KEGG enrichment of DEGs in (D) MSPms-VS-MPms, (E) MSTds-VS-MTds, and (F) MSMs-VS-MMs. The dot size indicates the number of enriched differentially expressed genes in each pathway, and the different color of the dot represents the $p$-value of each pathway. The top 20 enriched GO terms and KEGG enrichments ranked by $p$-values are shown. MS—Male sterile line; M-Maintainer line; Pms—pollen mother cell stage; Tds-tetrad stage; Ms—mononuclear stage.

\subsection{Identification and Enrichment Analyses of Cis-Target Genes of IncRNAs}

To identify potential cis-target genes (mRNAs within a $20 \mathrm{kbp}$ window upstream or downstream of the lncRNAs), Pearson's correlation matrix was calculated, in accordance with the criteria of |Pearson's correlation| > 0.6, 523 mRNAs, 592 mRNAs, and 690 mRNAs, corresponding to 366 lncRNAs, 397 lncRNAs, and 379 lncRNAs, were considered cis-target genes at the Pms stage, Tds stage, and Ms stage, respectively, and details of the chromosomal mapping of lncRNA are given in the Supplementary Section (Table S4a-c). Hierarchical clustering of DE lncRNAs and their regulated mRNA were differentially expressed at different stages between C2P5B and C2P5A (Figure 4A,B).

At the Pms stage, the GO enrichment analysis showed that 523 cis-target genes of lncRNAs were significantly enriched for 10 processes, such as NADH dehydrogenase (ubiquinone) activity, oxidoreductase activity, and cytoplasm (Figure 5A and Table S5a). The cis-target genes of these lncRNA were significantly enriched for the 12 KEGG pathways, and the pathways mainly included fatty acid biosynthesis, flavonoid biosynthesis, glutathione metabolism, MAPK signaling pathway to the plant, oxidative phosphorylation, and ubiquinone and other terpenoid quinone biosynthesis (Figure 6A and Table S5b). 

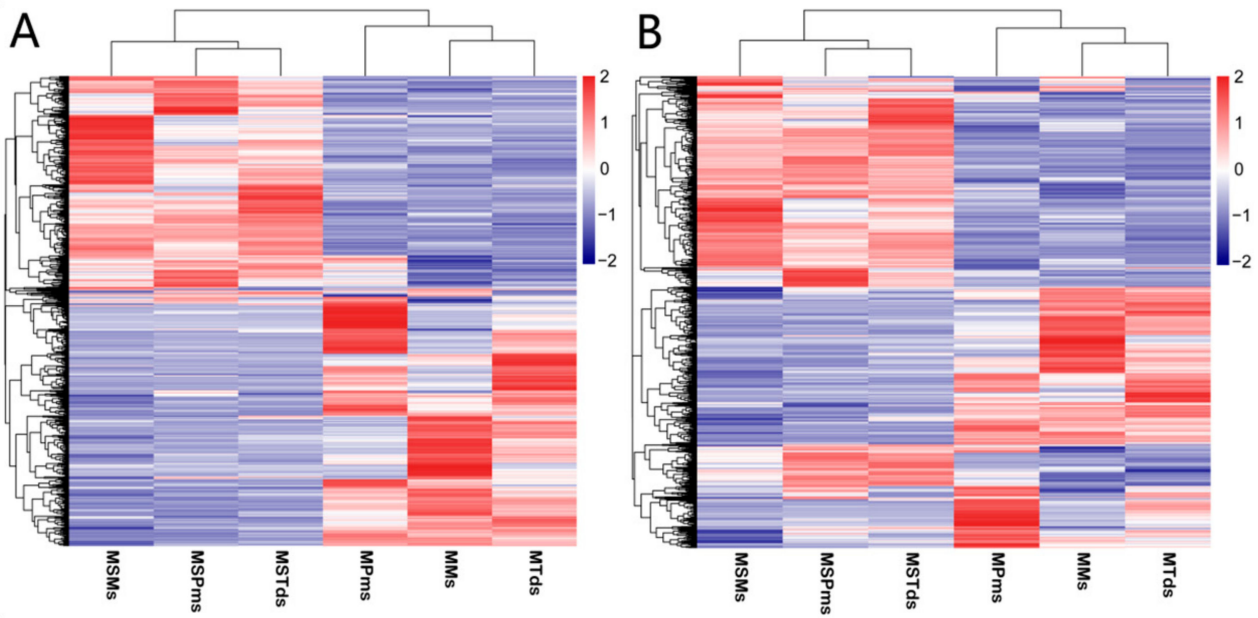

Figure 4. (A) Hierarchical clustering of lncRNAs differentially expressed at different stages in the development of cotton. (B) Hierarchical clustering of cis- target genes of lncRNAs differentially expressed at different stages in cotton development. Data are expressed as FPKM. Red: relatively high expression; Blue: relatively low expression. The bar code on the right represents the color scale of the $\log _{2}$ values. MS-Male sterile line; M-Maintainer line; Pms—-pollen mother cell stage; Tds—tetrad stage; Ms-mononuclear stage.

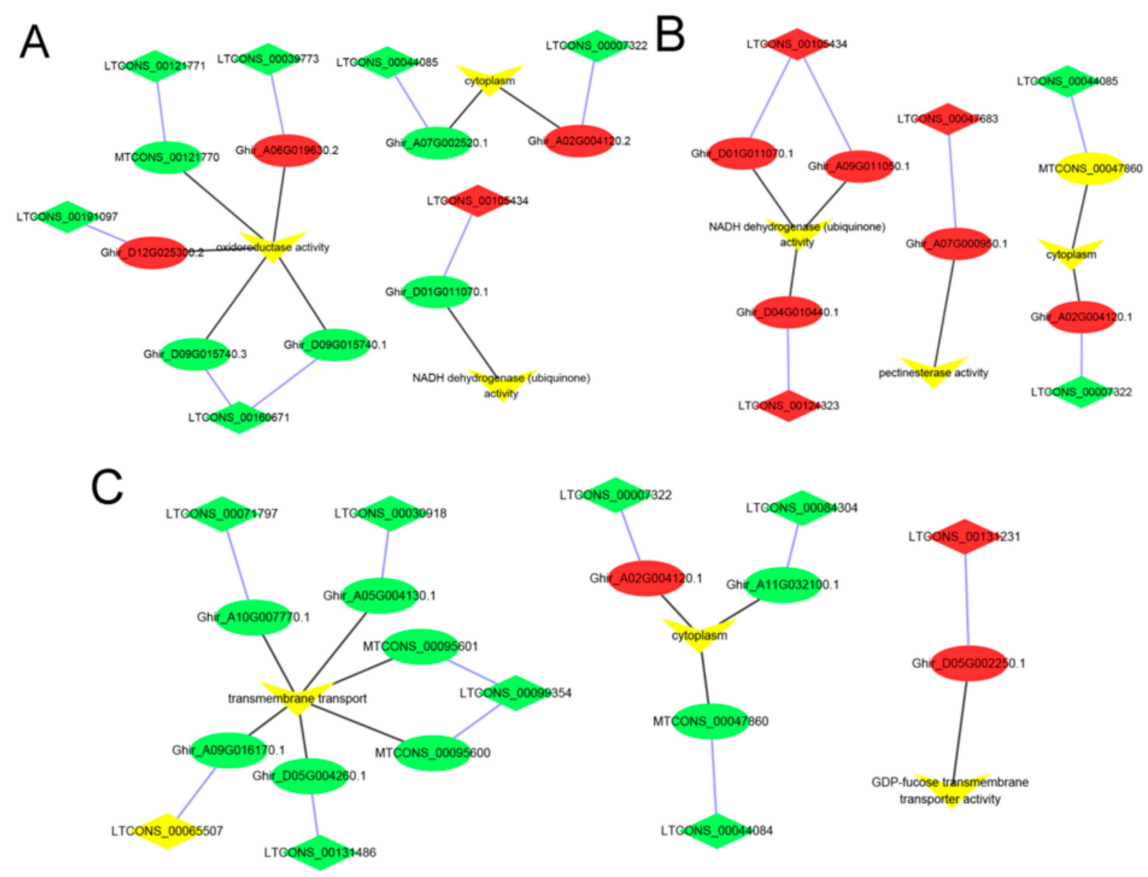

Figure 5. GO analysis enriched for lncRNA-potential cis-target genes of anther development between the cytoplasmic male sterility line C2P5A, and the maintainer line C2P5B. (A) GO terms enriched for the Pms stage lncRNAs-mRNAs. (B) GO terms enriched for the Tds stage lncRNAs-mRNAs. (C) GO terms enriched for the Ms stage lncRNAs-mRNAs. The ellipse, diamond, and inverted triangle nodes represent the mRNAs (cis-target genes), lncRNAs, and GO terms, respectively. Red represents upregulated, green represents downregulated, and yellow represents the enrichment of the GO terms. The blue edges and black edges show regulatory interactions among cis-target genes and lncRNAs, genes (cis-target genes and lncRNAs) and GO terms, respectively. 


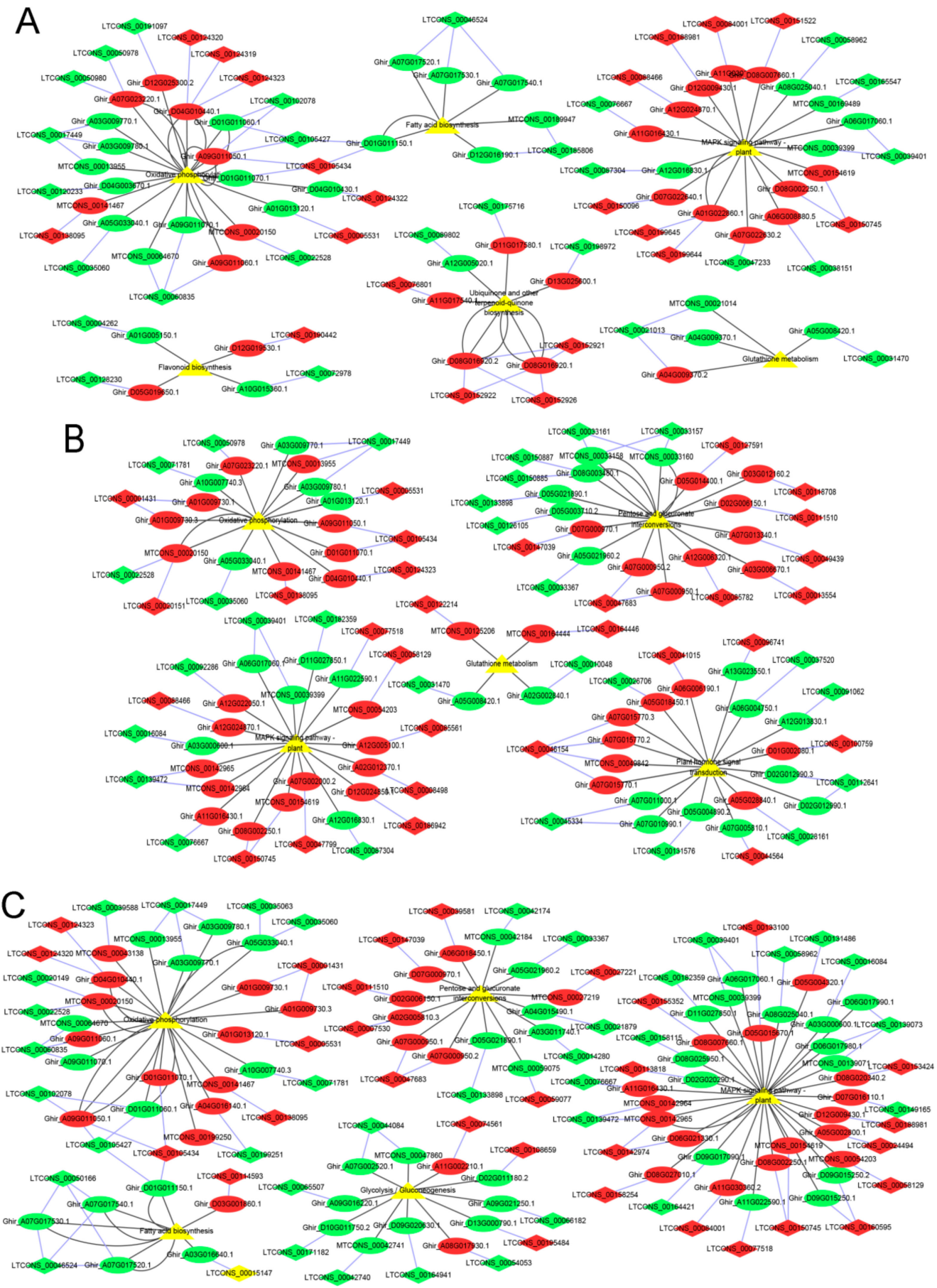

Figure 6. Kyoto Encyclopedia of Genes and Genomes pathways (KEGG) enriched for lncRNA-potential cis-target genes of anther development, between the cytoplasmic male sterility line C2P5A, and the maintainer line C2P5B. (A) KEGG pathways enriched for the Pms stage lncRNAs-mRNAs. (B) KEGG pathways enriched for the Tds stage lncRNAs-mRNAs. (C) KEGG pathways enriched for the Ms stage lncRNAs-mRNAs. The ellipse, diamond, and triangle nodes represent the mRNAs (cis-target genes), lncRNAs, and pathway, respectively. Red represents upregulated, green represents downregulated, and yellow represents the enrichment of pathways. The blue edges showed interaction between the mRNA and lncRNA. The black edges showed regulatory interaction transcriptomes and pathways.

At the Tds stage, 592 cis-target genes of lncRNAs were significantly enriched for NADH dehydrogenase (ubiquinone) activity, cytoplasm, and pectinesterase activity (Figure 5B and Table S5c). 
Of these cis-target genes, they were significantly enriched for 11 KEGG pathways, which mainly consisted of glutathione metabolism, MAPK signaling pathway to the plant, oxidative phosphorylation, pentose and glucuronate interconversions, and plant hormone signal transduction (Figure 6B and Table S5d).

At the Ms stage, 690 cis-target genes of lncRNAs were significantly enriched for 12 GO terms and 10 KEGG pathways, and the GO terms mainly included the cytoplasm and GDP-fucose transmembrane transporter activity (Figure 5C and Table S5e). The significant KEGG pathways mainly consisted of fatty acid biosynthesis, MAPK signaling pathway to the plant, oxidative phosphorylation, pentose and glucuronate interconversions, and phenylalanine metabolism (Figure 6C and Table S5f).

In this paper, the GO annotation and KEGG pathway enrichment were performed for DE mRNAs and DE lncRNAs-cis-target genes. The results show that these terms and pathways play a crucial role in response to the cotton anther abortion of the CMS line C2P5A.

\subsection{Validation of RNA-Sequencing (RNA-Seq) by Real-Time Quantitative PCR (RT-qPCR)}

RT-qPCR verified the expression level of the selected DE lncRNAs and the cis-target of the IncRNAs. In this paper, we randomly selected three, five, and four lncRNAs, corresponding to three, five, and five cis-target genes of lncRNAs at the Pms, Tds, and Ms stage of the anther development, respectively, between the CMS line C2P5A and the maintainer line C2P5B (Figure 7 and Table S6a-c). The specific primers were designed by Primer Premier 6.0 software (Table S7a-c). Pearson's coefficient was used to analyze the correlation between the qPCR and RNA-seq data. Overall, the expression level of 12 lncRNA DEGs and 13 cis-target genes of lncRNAs RT-qPCR was calculated using the $2^{-\Delta \Delta C t}$ method, which is basically consistent with the RNA-seq data (lncRNA, Figure S1A, correlation coefficient $=0.77$; cis-target genes of lncRNAs, Figure S2A, correlation coefficient $=0.58$ ). The lncRNAs at the Pms, Tds, and Ms stage of the anther development for the RT-qPCR and RNA-seq correlation coefficient were $0.78,0.72$, and 0.92 , respectively (Figure S1B-D). The cis-target gene of lncRNA, at the Pms, Tds, and Ms stage of the anther development RT-qPCR and RNA-seq correlation coefficient were $0.70,0.91$, and 0.63 , respectively (Figure S2B-D). The results showed that our RNA-seq data were reliable and conducive for screening DEGs during anther development.
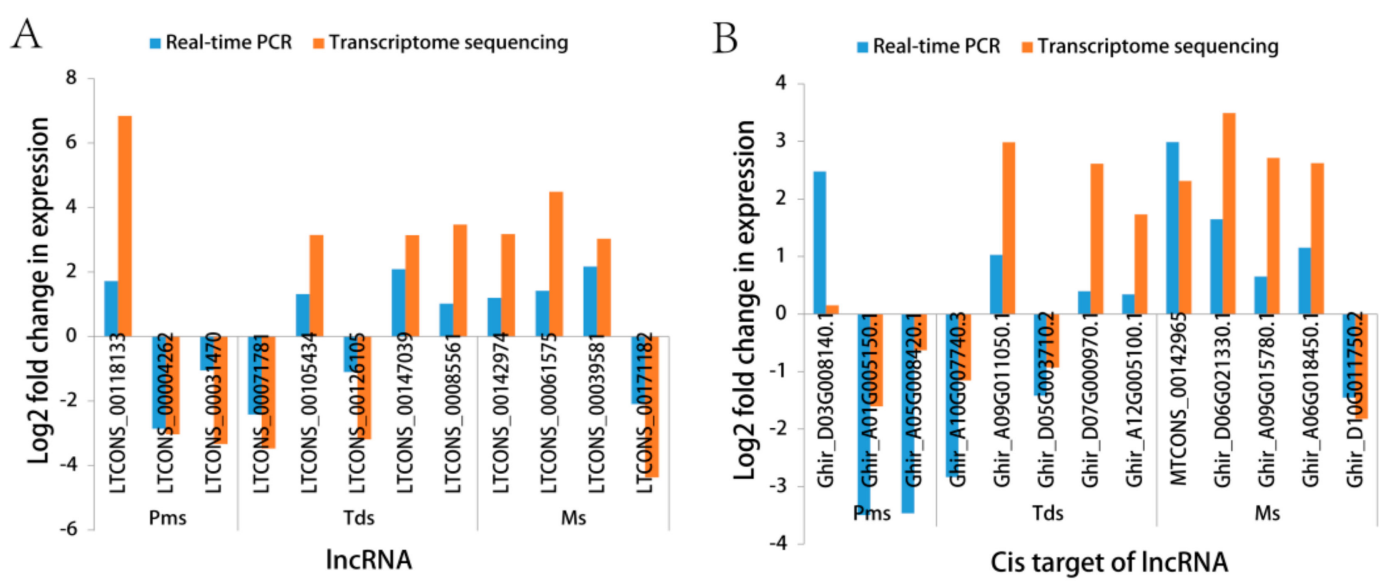

Figure 7. Quantitative PCR (RT-qPCR) confirmation of the RNA-sequencing (RNA-Seq) expression profiles at different stages of anther development of cotton, between the cytoplasmic male sterility line C2P5A and the maintainer line C2P5B. (A) The expression levels of 12 differentially expressed long noncoding RNAs. (B) The expression levels of 13 cis-target genes of lncRNAs corresponding to (A) lncRNAs, respectively. Of these lncRNAs, LTCONS_00142974 regulated two cis-target genes, MTCONS_00142965, Ghir_D06G021330.1. GhACT4-actin was used as a reference gene for normalization in our experiments. The fold change from the RT-qPCR was calculated using the $2^{-\Delta \Delta C t}$ method. 


\section{Discussion}

\subsection{Transcriptome mRNA in the Anther Development of Cotton}

Anther development is a complex process, with numerous genes and various metabolic pathways being involved. In model plants like Arabidopsis and rice, many genes that regulate the fate of somatic germ cells and the differentiation of the anther wall, as well as control the degradation of tapetum cells and microspore development during anther development, have been identified [49-51]. In cotton, the GhACS1 gene encodes an acyl-CoA synthetase, which is essential for normal microspore development, and highly expressed in sporogenous cells, pollen mother cells, microspores, and tapetum cells [52]. The actin-depolymerizing factor (GhADF7) gene may play an important role in pollen development and germination, and its transcript expression reaches a peak at flowering [53]. GhMYB24 encodes the MYB-like transcription factor that regulates the development of the tapetum [54].

Carbohydrates provide energy and nutrients for anther development; the sink strength of anthers is the highest in the early stages of anther development, which is intensively energy-demanding [55] and, thus, abnormal carbohydrate metabolism can significantly damage pollen development and cause male sterility [56,57]. Sugar is converted to starch so as to ensure energy preservation for pollen maturation and bud germination [57]. Zhang and his team studied the sterility of the male-sterile line 1355A of cotton, and found soluble sugar and fatty acid metabolism to play a central role in anther development. In the male sterile line 1355A, soluble sugars are decreased, and fatty acid synthesis is key for regulating normal pollen hydration and the primary component of sporopollenin, which can protect pollen from various stresses and is crucial for pollen grain development and male sterility $[58,59]$. High rates of glucose metabolism may promote fatty acid synthesis in order to promote the anther development of cotton [59]. Researchers studying cotton (Gossypium hirsutum) anthers under a high temperature (HT) and normal temperature (NT) indicated that HT disturbs sugar and ROS metabolism by disrupting DNA methylation, leading to microspore sterility [60]. Flavonoids play an important role in the formation of pollen exine formation, pollen germination, and the fertility of several plants that are key branch-point genes during tetrad and uninucleate microspore periods. Some researchers who studied genic male sterility (GMS) mutant anther development indicated that flavonoid metabolism is initially activated at the tetrad stage, then suppressed at the uninucleate microspore stage, leading to male sterility and the absence of flavonoids in mature stamens [61]. By using digital gene expression (DGE), some researchers identified many of the key genes that are required for cotton anther development and those that are mainly associated with sucrose and starch metabolism, the pentose phosphate pathway, glycolysis, and flavonoid metabolism [61,62].

Hormone signal transduction plays an important role in anther development, such as that involving ethylene, gibberellic acid (GA), and abscisic acid (ABA); higher amounts of ethylene may directly lead to the premature degeneration of the tapetal layer in GMS mutant anthers [59]. GA can accelerate flowering and promote the development of female flowers; conversely, GA deficiency can cause the abnormal development of anthers $[63,64]$. In addition, high levels of ABA delayed endosperm differentiation and the lack of endosperm leads to difficulties in germination [65]. Some researchers have shown that the MAPK signaling pathway plays a key role in the differentiation, proliferation, and apoptosis of cells [66].

In this research, we identified 17,897 known DE mRNAs at three stages of anther development, between the CMS line C2P5A and the maintainer line C2P5B (Table S1e). These DE mRNAs underwent GO enrichment and KEGG enriched pathway analyses, which showed that the significant terms were mostly enriched in malate synthase activity, catalytic activity, carbohydrate metabolic process, oxidoreductase activity, oxygen carrier activity, and glucan metabolic process (Figure $3 \mathrm{~A}-\mathrm{C}$ and Table $\mathrm{S} 3 \mathrm{a}, \mathrm{c}, \mathrm{e})$, and the significantly enriched pathways were flavonoid biosynthesis, plant hormone signal transduction, fatty acid metabolism, starch and sucrose metabolism, and MAPK signaling pathways in plants (Figure 3D-F and Table S3b,d,f). Compared to the same anther developmental stage of the maintainer line $\mathrm{C} 2 \mathrm{P} 5 \mathrm{~B}$, there were many key genes with an abnormal expression pattern in the CMS 
line C2P5A, which indicated that the abortion of cotton C2P5A was related to the abnormal metabolic pathway of the abnormally expressed gene regulating anther development.

\subsection{IncRNA in the Anther Development of Cotton and Predicted Functions}

The genomes of the eukaryotes are universally transcribed. Some RNAs are encoded into proteins, and other thousands of lncRNAs regulate key molecular and biological processes [46]. Until now, some of the best-studied mammalian lncRNAs have associated the dysregulation of lncRNAs with reproduction, including germ cell specification, early embryo implantation and development, and reproductive hormone regulation $[67,68]$, but the involvement of plant lncRNAs in reproduction is still poorly understood. In this study, we identified that the expression profiles of mRNA and lncRNA were related to cotton anther male sterility and were differentially expressed at different anther development periods. When further analyzed, the interaction network between the lncRNAs and mRNAs based on expression profiles shows that these transcriptomes may play crucial roles in the anther development of cotton.

In a previous study, we used a chromatin state map approach and RNA-seq to identify what is typically co-expressed with lncRNAs and involved in regulating their neighboring mRNA [69,70]. In animals, many studies have shown that lncRNAs directly regulate their neighboring genes in a cis-acting manner, such as IncRNA-TCONS_00175604 cis-action in dairy goat [71], lncRNA-NEF cis-regulating neighbor gene FOXA2 in mice [72], lncRNA-Six1 cis-regulating neighbor gene Six1 in chicken [73], IncRNA-Malat1 in mouse [74], lncRNA-TBILA cis-regulating HGAL in humans [75], and lncRNA-Jpx via both trans- and cis-Xist expression in mice [76].

In plants, lncRNAs regulate many molecular functions and biological processes in various ways. LncRNAs can pair with cis- or trans-transcripts, translation inhibition, and gene silencing [46]. The Arabidopsis noncoding RNA HID1 promotes photomorphogenesis in continuous red light (CR) and acts through PIF3 [77]. During vernalization in Arabidopsis, antisense lncRNA COOLAIR is associated with the FLOWERING LOCUS C (FLC) locus and switches of chromatin states during epigenetic regulation; COOLAIR participates in the autonomous pathway and controls the flowering time [8,78,79]; and intronic sense lncRNA COLDAIR acts as a scaffold RNA to recruit the PRC2 complex and establish FLC epigenetic silencing, and mediates FRIGIDA (FRI) degradation [8,80,81].

Natural antisense lncRNAs TWISTED LEAF(TL) play a cis-regulatory role in OsMYB60 expression and for maintaining leaf blade flattening [82]. Overexpressing lncRNA LAIR upregulates the expression of the neighbor gene LRK (leucine-rich repeat receptor kinase) cluster, which increases rice grain yield [83]. LncRNA1459 was knocked out by CRISPR/Cas9 altered tomato fruit in ripening; in these mutants, the ethylene production and lycopene accumulation were largely repressed [84].

In cotton, previous studies have shown that lncRNAs XLOC_063105-CotAD_37096 and XLOC_115463-CotAD_12502 probably function in cis-regulating responses to drought stress [32]. One lncRNA, TCONS_00061835-Gh_D06G1439 (GhMYB-like), regulates cotton fiber development [36] by complementary base pairing with the protein-coding gene, lncRNA, and the circRNA complex network, demonstrating that some lncRNAs are involved in biotic and abiotic stresses [31,33,34].

In this study, we sought the cis-target genes within a radius of $20 \mathrm{kbp}$ for the lncRNAs, and identified 865 lncRNAs that might exert their functions through the predicted cis-target genes' 1172 mRNAs (Table S4d). The predicted cis-target genes underwent GO enrichment and KEGG pathway analyses, and the results provided ideas for future research. The significant GO terms were enriched in NADH dehydrogenase (ubiquinone) activity, oxidoreductase activity, photosynthetic electron transport, cytoplasm, and pectinesterase activity (Figure 5A-C). NADH dehydrogenase (ubiquinone), oxidoreductase, and photosynthetic electron transport play a crucial role in energy production and conversion, in which the proteins are components in the mitochondrial respiratory chain. This showed that mitochondrial respiratory-related enzymes play an important role in the regulation of the CMS line C2P5A, and this result is consistent with a previous study in CMS plants [85-87]. Pectinesterase is a wall-degrading enzyme, and abnormal expression may lead to the disruption of the 
cell wall structure in the CMS line C2P5A; a previous study demonstrated that pectinesterase plays a major role in the plant [88]. In our research, the GO of the cytoplasm was significant, showing that its abnormality may be one of the key factors leading to abortion in the CMS line C2P5A compared to the maintainer line C2P5B.

Significant KEGG pathways were primarily enriched for several processes, such as fatty acid biosynthesis, flavonoid biosynthesis, glutathione metabolism, MAPK signaling pathway to the plant, oxidative phosphorylation, ubiquinone and other terpenoid quinone biosynthesis, pentose and glucuronate interconversions, and plant hormone signal transduction (Figure 6A-C). Previous studies show that these metabolism pathways play a vital role during anther development in flowering plants, in which a disturbed metabolism pathway seriously leads to the impairment of anther development, and causes male sterility $[55,57,61,62,85,89]$. This study showed that some lncRNAs and mRNAs might play important roles in anther development, such as cis-target gene Ghir_A09G011050.1 of LTCONS_00105434 through GO:0008137 (NADH dehydrogenase (ubiquinone) activity) and ko00190 (oxidative phosphorylation); cis-target gene Ghir_A01G005150.1 of LTCONS_00004262 through GO:0016491 (oxidoreductase activity) and ko00941 (flavonoid biosynthesis); cis-target gene Ghir_D05G003710.2 of LTCONS_00126105 through GO:0005975 (carbohydrate metabolic process) and ko00040 (pentose and glucuronate interconversions); cis-target gene Ghir_A03G016640.1 of LTCONS_00085561 through GO:0016790 (catalytic activity) and ko00061 (fatty acid biosynthesis); and cis-target gene Ghir_A12G005100.1 of LTCONS_00085561 through GO:0005524 (ATP binding) and ko04016 (MAPK signaling pathway to the plant).

\section{Conclusions}

In this study, we carried out transcriptome studies to identify DE mRNA and lncRNA and performed GO annotation and pathway enrichment analysis on the potential cis-target genes of these DE lncRNAs, showing their important roles in the regulation of anther development in cotton. Of these DE genes, LTCONS_00105434, LTCONS_00004262, LTCONS_00126105, LTCONS_00085561, and LTCONS_00085561 correspond to the cis-target genes Ghir_A09G011050.1, Ghir_A01G005150.1, Ghir_D05G003710.2, Ghir_A03G016640.1, and Ghir_A12G005100.1, respectively, which participate in anther development. This research provides us with a better perspective of the molecular regulation of the anther development of CMS line C2P5A in cotton.

Supplementary Materials: The following are available online at http:/www.mdpi.com/2073-4425/10/12/947/s1. Figure S1: LncRNA RT-PCR correlation RNA-seq; Figure S2: cis-target gene of lncRNA RT-PCR correlation RNA-seq; Table S1: New identified DE mRNAs at three stages of cotton anther development between the CMS line and the maintainer line; Table S2: Newly identified DE lncRNAs at three stages of cotton anther development between the CMS line and the maintainer line; Table S3: Enriched GO and KEGG terms for DE mRNA in three stages of anther development between the CMS line and the maintainer line; Table S4: cis-target gene of lncRNAs in three stages of anther development between the CMS line and the maintainer line; Table S5: Enriched GO and KEGG terms for cis-taget genes of lncRNA in three stages of anther development between the CMS line and the maintainer line; Table S6: DEGs of RNA-sequencing data at three stages of the anther development between the CMS line and the maintainer line; Table S7: Genes and qPCR primer sequences.

Author Contributions: Q.W. conceived the experiments and revised the paper; Q.C. conceived and designed the research; N.D., C.W., Y.Z., R.S., and T.D. participated in material collection; R.Z. provided the materials and guided the experiment; T.Q. revised the manuscript; Y.L. performed the experiments and wrote the manuscript.

Funding: This study was supported by the National Key Research and Development Program of Thirteenth (project 2016YFD0101413), the Key Scientific Research Project of Colleges of Henan Province (project 105020216010/010), the National Natural Science Foundation of China (project 31170263), the Major Science and Technology Projects in Henan Province (project 161100510100), and the Science and Technique Foundation Project of Henan Province (project 182102110003).

Acknowledgments: We thank Ruiyang Zhou (College of Agriculture, Guangxi University) for providing the cytoplasmic male sterility C2P5A and the maintainer line C2P5B material.

Conflicts of Interest: The authors declare no conflict of interest. 


\section{References}

1. Derrien, T.; Guigo, R.; Johnson, R. The Long Non-Coding RNAs: A New (P)layer in the "Dark Matter". Front. Genet. 2011, 2, 107. [CrossRef]

2. Ma, X.; Shao, C.; Jin, Y.; Wang, H.; Meng, Y. Long non-coding RNAs: A novel endogenous source for the generation of Dicer-like 1-dependent small RNAs in Arabidopsis thaliana. Rna Biol. 2014, 11, 373-390. [CrossRef]

3. Murakami, K. Non-coding RNAs and hypertension-unveiling unexpected mechanisms of hypertension by the dark matter of the genome. Curr. Hypertens. Rev. 2015, 11, 80-90. [CrossRef] [PubMed]

4. Chekanova, J.A. Long non-coding RNAs and their functions in plants. Curr. Opin. Plant. Biol. 2015, 27, 207-216. [CrossRef] [PubMed]

5. Wang, M.; Yuan, D.; Tu, L.; Gao, W.; He, Y.; Hu, H.; Wang, P.; Liu, N.; Lindsey, K.; Zhang, X. Long noncoding RNAs and their proposed functions in fibre development of cotton (Gossypium spp.). New Phytol. 2015, 207, 1181-1197. [CrossRef] [PubMed]

6. Wang, R.; Zou, J.; Meng, J.; Wang, J. Integrative analysis of genome-wide lncRNA and mRNA expression in newly synthesized Brassica hexaploids. Ecol. Evol. 2018, 8, 6034-6052. [CrossRef] [PubMed]

7. Bhan, A.; Mandal, S.S. Long noncoding RNAs: Emerging stars in gene regulation, epigenetics and human disease. Chemmedchem. 2014, 9, 1932-1956. [CrossRef]

8. Shafiq, S.; Li, J.; Sun, Q. Functions of plants long non-coding RNAs. BBA Gene Regul. Mech. 2016, 1859, 155-162. [CrossRef]

9. Lu, Z.; Xia, X.; Jiang, B.; Ma, K.; Zhu, L.; Wang, L.; Jin, B. Identification and characterization of novel lncRNAs in Arabidopsis thaliana. Biochem Biophys Res. Commun. 2017, 488, 348-354. [CrossRef]

10. Zhang, Y.; Liao, J.; Li, Z.; Yu, Y.; Zhang, J.; Li, Q.; Qu, L.; Shu, W.; Chen, Y. Genome-wide screening and functional analysis identify a large number of long noncoding RNAs involved in the sexual reproduction of rice. Genome Biol. 2014, 15, 512. [CrossRef]

11. Mattick, J.S.; Rinn, J.L. Discovery and annotation of long noncoding RNAs. Nat. Struct. Mol. Biol. 2015, 22, 5-7. [CrossRef] [PubMed]

12. Wang, M.; Zhao, W.; Gao, L.; Zhao, L. Genome-wide profiling of long non-coding RNAs from tomato and a comparison with mRNAs associated with the regulation of fruit ripening. BMC Plant. Biol. 2018, 18, 75. [CrossRef] [PubMed]

13. Wang, A.; Hu, J.; Gao, C.; Chen, G.; Wang, B.; Lin, C.; Song, L.; Ding, Y.; Zhou, G. Genome-wide analysis of long non-coding RNAs unveils the regulatory roles in the heat tolerance of Chinese cabbage (Brassica rapa ssp.chinensis). Sci. Rep. 2019, 9, 5002. [CrossRef] [PubMed]

14. Xin, M.; Wang, Y.; Yao, Y.; Song, N.; Hu, Z.; Qin, D.; Xie, C.; Peng, H.; Ni, Z.; Sun, Q. Identification and characterization of wheat long non-protein coding RNAs responsive to powdery mildew infection and heat stress by using microarray analysis and SBS sequencing. BMC Plant. Biol. 2011, 11, 61. [CrossRef]

15. Seo, J.S.; Sun, H.X.; Park, B.S.; Huang, C.H.; Yeh, S.D.; Jung, C.; Chua, N.H. ELF18-INDUCED LONG-NONCODING RNA Associates with Mediator to Enhance Expression of Innate Immune Response Genes in Arabidopsis. Plant. Cell 2017, 29, 1024-1038. [CrossRef]

16. Cui, J.; Luan, Y.; Jiang, N.; Bao, H.; Meng, J. Comparative transcriptome analysis between resistant and susceptible tomato allows the identification of lncRNA16397 conferring resistance to Phytophthora infestans by co-expressing glutaredoxin. Plant. J. 2017, 89, 577-589. [CrossRef]

17. Chen, L.; Shi, S.; Jiang, N.; Khanzada, H.; Wassan, G.M.; Zhu, C.; Peng, X.; Xu, J.; Chen, Y.; Yu, Q.; et al. Genome-wide analysis of long non-coding RNAs affecting roots development at an early stage in the rice response to cadmium stress. BMC Genom. 2018, 19, 460. [CrossRef]

18. Burleigh, S.H.; Harrison, M.J. A novel gene whose expression in Medicago truncatula roots is suppressed in response to colonization by vesicular-arbuscular mycorrhizal (VAM) fungi and to phosphate nutrition. Plant. Mol. Biol. 1997, 34, 199-208. [CrossRef]

19. Yuan, J.; Zhang, Y.; Dong, J.; Sun, Y.; Lim, B.L.; Liu, D.; Lu, Z.J. Systematic characterization of novel lncRNAs responding to phosphate starvation in Arabidopsis thaliana. BMC Genom. 2016, 17, 655. [CrossRef]

20. Chen, M.; Wang, C.; Bao, H.; Chen, H.; Wang, Y. Genome-wide identification and characterization of novel lncRNAs in Populus under nitrogen deficiency. Mol. Genet. Genom. 2016, 291, 1663-1680. [CrossRef] 
21. Ding, J.; Lu, Q.; Ouyang, Y.; Mao, H.; Zhang, P.; Yao, J.; Xu, C.; Li, X.; Xiao, J.; Zhang, Q. A long noncoding RNA regulates photoperiod-sensitive male sterility, an essential component of hybrid rice. Proc. Natl Acad. Sci. USA 2012, 109, 2654-2659. [CrossRef] [PubMed]

22. Guo, J.; Liu, Y.G. Long non-coding RNAs play an important role in regulating photoperiod- and temperature-sensitive male sterility in rice. Sci. China Life Sci. 2017, 60, 443-444. [CrossRef] [PubMed]

23. Kang, C.; Liu, Z. Global identification and analysis of long non-coding RNAs in diploid strawberry Fragaria vesca during flower and fruit development. BMC Genom. 2015, 16, 815. [CrossRef] [PubMed]

24. Wang, C.Y.; Liu, S.R.; Zhang, X.Y.; Ma, Y.J.; Hu, C.G.; Zhang, J.Z. Genome-wide screening and characterization of long non-coding RNAs involved in flowering development of trifoliate orange (Poncirus trifoliata L. Raf.). Sci Rep. 2017, 7, 43226. [CrossRef] [PubMed]

25. Wang, K.; Wang, Z.; Li, F.; Ye, W.; Wang, J.; Song, G.; Yue, Z.; Cong, L.; Shang, H.; Zhu, S.; et al. The draft genome of a diploid cotton Gossypium raimondii. Nat. Genet. 2012, 44, 1098-1103. [CrossRef]

26. Li, F.; Fan, G.; Wang, K.; Sun, F.; Yuan, Y.; Song, G.; Li, Q.; Ma, Z.; Lu, C.; Zou, C.; et al. Genome sequence of the cultivated cotton Gossypium arboreum. Nat. Genet. 2014, 3, 567-572. [CrossRef] [PubMed]

27. Zhang, T.; Hu, Y.; Jiang, W.; Fang, L.; Guan, X.; Chen, J.; Zhang, J.; Saski, C.A.; Scheffler, B.E.; Stelly, D.M.; et al. Sequencing of allotetraploid cotton (Gossypium hirsutum L. acc. TM-1) provides a resource for fiber improvement. Nat. Biotechnol. 2015, 33, 531-537. [CrossRef]

28. Zhu, Y.X.; Li, F.G. The Gossypium raimondii Genome, a Huge Leap Forward in Cotton Genomics. J. Integr. Plant. Biol. 2013, 55, 570-571. [CrossRef]

29. Wang, M.; Tu, L.; Yuan, D.; Zhu, D.; Shen, C.; Li, J.; Liu, F.; Pei, L.; Wang, P.; Zhao, G.; et al. Reference genome sequences of two cultivated allotetraploid cottons, Gossypium hirsutum and Gossypium barbadense. Nat. Genet. 2019, 51, 224-229. [CrossRef]

30. Hu, Y.; Chen, J.; Fang, L.; Zhang, Z.; Ma, W.; Niu, Y.; Ju, L.; Deng, J.; Zhao, T.; Lian, J.; et al. Gossypium barbadense and Gossypium hirsutum genomes provide insights into the origin and evolution of allotetraploid cotton. Nat. Genet. 2019, 51, 739-748. [CrossRef]

31. Zhang, L.; Wang, M.; Li, N.; Wang, H.; Qiu, P.; Pei, L.; Xu, Z.; Wang, T.; Gao, E.; Liu, J.; et al. Long noncoding RNAs involve in resistance to Verticillium dahliae, a fungal disease in cotton. Plant. Biotechnol. J. 2018, 16, 1172-1185. [CrossRef] [PubMed]

32. Lu, X.; Chen, X.; Mu, M.; Wang, J.; Wang, X.; Wang, D.; Yin, Z.; Fan, W.; Wang, S.; Guo, L. Genome-Wide Analysis of Long Noncoding RNAs and Their Responses to Drought Stress in Cotton (Gossypium hirsutum L.). PLoS ONE 2016, 11, e0156723. [CrossRef] [PubMed]

33. Deng, F.; Zhang, X.; Wang, W.; Yuan, R.; Shen, F. Identification of Gossypium hirsutum long non-coding RNAs (lncRNAs) under salt stress. BMC Plant. Biol. 2018, 18, 23-37. [CrossRef] [PubMed]

34. Song, Y.; Li, L.; Yang, Z.; Zhao, G.; Zhang, X.; Wang, L.; Zheng, L.; Zhuo, F.; Yin, H.; Ge, X.; et al. Target of Rapamycin (TOR) Regulates the Expression of lncRNAs in Response to Abiotic Stresses in Cotton. Front. Genet. 2018, 9, 690. [CrossRef]

35. Zou, C.; Wang, Q.; Lu, C.; Yang, W.; Zhang, Y.; Cheng, H.; Feng, X.; Prosper, M.A.; Song, G. Transcriptome analysis reveals long noncoding RNAs involved in fiber development in cotton (Gossypium arboreum). Sci. China Life Sci. 2016, 59, 164-171. [CrossRef]

36. Hu, H.; Wang, M.; Ding, Y.; Zhu, S.; Zhao, G.; Tu, L.; Zhang, X. Transcriptomic repertoires depict the initiation of lint and fuzz fibres in cotton (Gossypium hirsutum L.). Plant. Biotechnol J. 2018, 16, 1002-1012. [CrossRef]

37. Kim, D.; Langmead, B.; Salzberg, S.L. HISAT: A fast spliced aligner with low memory requirements. Nat. Methods 2015, 12, 357-360. [CrossRef]

38. Finn, R.D.; Coggill, P.; Eberhardt, R.Y.; Eddy, S.R.; Mistry, J.; Mitchell, A.L.; Potter, S.C.; Punta, M.; Qureshi, M.; Sangrador-Vegas, A.; et al. The Pfam protein families database: Towards a more sustainable future. Nucleic Acids Res. 2016, 44, 279-285. [CrossRef]

39. Pertea, M.; Pertea, G.M.; Antonescu, C.M.; Chang, T.C.; Mendell, J.T.; Salzberg, S.L. StringTie enables improved reconstruction of a transcriptome from RNA-seq reads. Nat. Biotechnol. 2015, 33, 290-295. [CrossRef]

40. Trapnell, C.; Williams, B.A.; Pertea, G.; Mortazavi, A.; Kwan, G.; van Baren, M.J.; Salzberg, S.L.; Wold, B.J.; Pachter, L. Transcript assembly and quantification by RNA-Seq reveals unannotated transcripts and isoform switching during cell differentiation. Nat. Biotechnol. 2010, 28, 511-515. [CrossRef] 
41. Li, B.; Dewey, C.N. RSEM: Accurate transcript quantification from RNA-Seq data with or without a reference genome. BMC Bioinf. 2011, 12, 323. [CrossRef] [PubMed]

42. Trapnell, C.; Roberts, A.; Goff, L.; Pertea, G.; Kim, D.; Kelley, D.R.; Pimentel, H.; Salzberg, S.L.; Rinn, J.L.; Pachter, L. Differential gene and transcript expression analysis of RNA-seq experiments with TopHat and Cufflinks. Nat. Protoc. 2012, 7, 562-578. [CrossRef] [PubMed]

43. Kong, L.; Zhang, Y.; Ye, Z.; Liu, X.; Zhao, S.; Wei, L.; Gao, G. CPC: Assess the protein-coding potential of transcripts using sequence features and support vector machine. Nucleic Acids Res. 2007, 35, W345. [CrossRef] [PubMed]

44. Sun, L.; Luo, H.; Bu, D.; Zhao, G.; Yu, K.; Zhang, C.; Liu, Y.; Chen, R.; Zhao, Y. Utilizing sequence intrinsic composition to classify protein-coding and long non-coding transcripts. Nucleic Acids Res. 2013, 41, e166. [CrossRef] [PubMed]

45. Wang, L.; Feng, Z.; Wang, X.; Wang, X.; Zhang, X. DEGseq: An R package for identifying differentially expressed genes from RNA-seq data. Bioinformatics 2010, 26, 136-138. [CrossRef]

46. Wang, H.V.; Chekanova, J.A. Long Noncoding RNAs in Plants. Adv. Exp. Med. Biol. 2017, 1008, $133-154$.

47. Carlin, D.E.; Demchak, B.; Pratt, D.; Sage, E.; Ideker, T. Network propagation in the cytoscape cyberinfrastructure. PLoS Comput. Biol. 2017, 13, e1005598. [CrossRef]

48. Livak, K.J.; Schmittgen, T.D. Analysis of relative gene expression data using real-time quantitative PCR and the 2(-Delta Delta C(T)) Method. Methods 2001, 25, 402-408. [CrossRef]

49. Shu-Lan, Y.; Li-Fen, X.; Hui-Zhu, M.; Ching San, P.; Wei-Cai, Y.; Lixi, J.; Venkatesan, S.; De, Y. Tapetum determinant1 is required for cell specialization in the Arabidopsis anther. Plant. Cell 2003, 15, 2792-2804.

50. Catherine, A.; Eugenia, R.; Valerie, H.; Erik, B.; Sacco, D.V. The Arabidopsis thaliana SOMATIC EMBRYOGENESIS RECEPTOR-LIKE KINASES1 and 2 control male sporogenesis. Plant. Cell 2005, 17, 3337-3349.

51. Fu, Z.; Yu, J.; Cheng, X.; Zong, X.; Xu, J.; Chen, M.; Li, Z.; Zhang, D.; Liang, W. The Rice Basic Helix-Loop-Helix Transcription Factor TDR INTERACTING PROTEIN2 Is a Central Switch in Early Anther Development. Plant. Cell 2014, 26, 1512-1524. [CrossRef] [PubMed]

52. Wang, X.L.; Li, X.B. The GhACS1 gene encodes an acyl-CoA synthetase which is essential for normal microsporogenesis in early anther development of cotton. Plant. J. 2009, 57, 473-486. [CrossRef] [PubMed]

53. Li, X.B.; Xu, D.; Wang, X.L.; Huang, G.Q.; Luo, J.; Li, D.D.; Zhang, Z.T.; Xu, W.L. Three cotton genes preferentially expressed in flower tissues encode actin-depolymerizing factors which are involved in F-actin dynamics in cells. J. Exp. Bot. 2010, 61, 41-53. [CrossRef] [PubMed]

54. Li, Y.; Jiang, J.; Du, M.L.; Li, L.; Wang, X.L.; Li, X.B. A cotton gene encoding MYB-like transcription factor is specifically expressed in pollen and is involved in regulation of late anther/pollen development. Plant. Cell Physiol. 2013, 54, 893-906. [CrossRef]

55. Oliver, S.N.; Dennis, E.S.; Dolferus, R. ABA regulates apoplastic sugar transport and is a potential signal for cold-induced pollen sterility in rice. Plant. Cell Physiol. 2007, 48, 1319-1330. [CrossRef]

56. Tang, H.; Song, Y.; Guo, J.; Wang, J.; Zhang, L.; Niu, N.; Ma, S.; Zhang, G.; Zhao, H. Physiological and metabolome changes during anther development in wheat (Triticum aestivum L.). Plant. Physiol Biochem. 2018, 132, 18-32.

57. Datta, R.; Chamusco, K.C.; Chourey, P.S. Starch biosynthesis during pollen maturation is associated with altered patterns of gene expression in maize. Plant. Physiol. 2002, 130, 1645-1656. [CrossRef]

58. Kim, S.S.; Douglas, C.J. Sporopollenin monomer biosynthesis in arabidopsis. J. Plant. Biol. 2013, 56, 1-6. [CrossRef]

59. Wu, Y.; Li, Y.; Li, Y.; Ma, Y.; Zhao, Y.; Wang, C.; Chi, H.; Chen, M.; Ding, Y.; Guo, X.; et al. Proteomic analysis reveals that sugar and fatty acid metabolisms play a central role in sterility of the male-sterile line 1355A of cotton. J. Biol Chem. 2019, 294, 7057-7067. [CrossRef]

60. Ma, Y.; Min, L.; Wang, M.; Wang, C.; Zhao, Y.; Li, Y.; Fang, Q.; Wu, Y.; Xie, S.; Ding, Y.; et al. Disrupted Genome Methylation in Response to High Temperature Has Distinct Affects on Microspore Abortion and Anther Indehiscence. Plant. Cell 2018, 30, 1387-1403. [CrossRef]

61. Wei, M.; Song, M.; Fan, S.; Yu, S. Transcriptomic analysis of differentially expressed genes during anther development in genetic male sterile and wild type cotton by digital gene-expression profiling. BMC Genom. 2013, 14, 97. [CrossRef] 
62. Zhang, Y.J.; Chen, J.; Liu, J.B.; Xia, M.X.; Wang, W.; Shen, F.F. Transcriptome Analysis of Early Anther Development of Cotton Revealed Male Sterility Genes for Major Metabolic Pathways. J. Plant. Growth Regul. 2014, 34, 223-232. [CrossRef]

63. Bagnall, D.J. Control of Flowering in Arabidopsis thaliana by Light, Vernalisation and Gibberellins. Funct. Plant. Biol. 1992, 19, 401-409. [CrossRef]

64. Thornsberry, J.M.; Goodman, M.M.; Doebley, J.; Kresovich, S.; Nielsen, D.; Buckler, E.S.T. Dwarf8 polymorphisms associate with variation in flowering time. Nat. Genet. 2001, 28, 286-289. [CrossRef] [PubMed]

65. Yan, X.; Tian, M.; Liu, F.; Wang, C.; Zhang, Y. Hormonal and morphological changes during seed development of Cypripedium japonicum. Protoplasma 2017, 254, 2315-2322. [CrossRef]

66. Chen, S.X.; Zhao, F.; Huang, X.J. MAPK signaling pathway and erectile dysfunction. Zhonghua Nan Ke Xue Natl. J. Androl. 2018, 24, 442-446.

67. Feng, X.; Li, F.; Wang, F.; Zhang, G.; Pang, J.; Ren, C.; Zhang, T.; Yang, H.; Wang, Z.; Zhang, Y. Genome-wide differential expression profiling of mRNAs and lncRNAs associated with prolificacy in Hu sheep. Biosci Rep. 2018, 38. [CrossRef] [PubMed]

68. Taylor, D.H.; Chu, E.T.; Spektor, R.; Soloway, P.D. Long non-coding RNA regulation of reproduction and development. Mol. Reprod Dev. 2015, 82, 932-956. [CrossRef]

69. Guttman, M.; Amit, I.; Garber, M.; French, C.; Lin, M.F.; Feldser, D.; Huarte, M.; Zuk, O.; Carey, B.W.; Cassady, J.P.; et al. Chromatin signature reveals over a thousand highly conserved large non-coding RNAs in mammals. Nature 2009, 458, 223-227. [CrossRef] [PubMed]

70. Cabili, M.N.; Trapnell, C.; Goff, L.; Koziol, M.; Tazon-Vega, B.; Regev, A.; Rinn, J.L. Integrative annotation of human large intergenic noncoding RNAs reveals global properties and specific subclasses. Genes Dev. 2011, 25, 1915-1927. [CrossRef]

71. Liu, X.; Zhang, L.; Cui, J.; Che, S.; Song, Y. The mRNA and lncRNA landscape of the non-pregnant endometrium during the oestrus cycle in dairy goat. Anim. Prod. Sci. 2019. [CrossRef]

72. $\quad$ Liang, W.C.; Ren, J.L.; Wong, C.W.; Chan, S.O.; Waye, M.M.; Fu, W.M.; Zhang, J.F. LncRNA-NEF antagonized epithelial to mesenchymal transition and cancer metastasis via cis-regulating FOXA2 and inactivating Wnt/beta-catenin signaling. Oncogene 2018, 37, 1445-1456. [CrossRef] [PubMed]

73. Cai, B.; Li, Z.; Ma, M.; Wang, Z.; Han, P.; Abdalla, B.A.; Nie, Q.; Zhang, X. LncRNA-Six1 Encodes a Micropeptide to Activate Six1 in Cis and Is Involved in Cell Proliferation and Muscle Growth. Front. Physiol. 2017, 8, 230. [CrossRef] [PubMed]

74. Bin, Z.; Gayatri, A.; Mao, Y.S.; Zsolt, L.; Gourab, B.; Xiaokun, X.; Booth, C.J.; Jie, W.; Chaolin, Z. The lncRNA Malat1 is dispensable for mouse development but its transcription plays a cis-regulatory role in the adult. Cell Rep. 2012, 2, 111-123.

75. Lu, Z.; Li, Y.; Che, Y.; Huang, J.; Sun, S.; Mao, S.; Lei, Y.; Li, N.; Sun, N.; He, J. The TGF $\beta$-induced lncRNA TBILA promotes non-small cell lung cancer progression in vitro and in vivo via cis-regulating HGAL and activating S100A7/JAB1 signaling. Cancer Lett. 2018, 432, 156-168. [CrossRef]

76. Carmona, S.; Lin, B.; Chou, T.; Arroyo, K.; Sun, S. LncRNA Jpx induces Xist expression in mice using both trans and cis mechanisms. PLoS Genet. 2018, 14, e1007378. [CrossRef]

77. Wang, Y.; Fan, X.; Lin, F.; He, G.; Terzaghi, W.; Zhu, D.; Deng, X.W. Arabidopsis noncoding RNA mediates control of photomorphogenesis by red light. Proc. Natl. Acad. Sci. USA 2014, 111, 10359-10364. [CrossRef]

78. Csorba, T.; Questa, J.I.; Sun, Q.; Dean, C. Antisense COOLAIR mediates the coordinated switching of chromatin states at FLC during vernalization. Proc. Natl. Acad. Sci. USA 2014, 111, 16160-16165. [CrossRef]

79. He, Y. Chromatin regulation of flowering. Trends Plant. Sci. 2012, 17, 556-562. [CrossRef]

80. Xiangyang, H.; Xiangxiang, K.; Chuntao, W.; Lan, M.; Jinjie, Z.; Jingjing, W.; Xiaoming, Z.; Loake, G.J.; Ticao, Z.; Jinling, H. Proteasome-mediated degradation of FRIGIDA modulates flowering time in Arabidopsis during vernalization. Plant. Cell 2014, 26, 4763-4781.

81. Heo, J.B.; Sung, S. Vernalization-mediated epigenetic silencing by a long intronic noncoding RNA. Science 2011, 331, 76-79. [CrossRef]

82. Liu, X.; Li, D.; Zhang, D.; Yin, D.; Zhao, Y.; Ji, C.; Zhao, X.; Li, X.; He, Q.; Chen, R.; et al. A novel antisense long noncoding RNA, TWISTED LEAF, maintains leaf blade flattening by regulating its associated sense R2R3-MYB gene in rice. New Phytol. 2018, 218, 774-788. [CrossRef] [PubMed] 
83. Wang, Y.; Luo, X.; Sun, F.; Hu, J.; Zha, X.; Su, W.; Yang, J. Overexpressing lncRNA LAIR increases grain yield and regulates neighbouring gene cluster expression in rice. Nat. Commun. 2018, 9, 3516. [CrossRef] [PubMed]

84. Li, R.; Fu, D.; Zhu, B.; Luo, Y.; Zhu, H. CRISPR/Cas9-mediated mutagenesis of lncRNA1459 alters tomato fruit ripening. Plant. J. 2018, 94, 513-524. [CrossRef] [PubMed]

85. Yang, Y.; Bao, S.; Zhou, X.; Liu, J.; Zhuang, Y. The key genes and pathways related to male sterility of eggplant revealed by comparative transcriptome analysis. BMC Plant. Biol. 2018, 18, 209. [CrossRef]

86. Nie, Z.; Zhao, T.; Yang, S.; Gai, J. Development of a cytoplasmic male-sterile line NJCMS4A for hybrid soybean production. Plant. Breed. 2017, 136, 516-525. [CrossRef]

87. Wang, K.; Gao, F.; Ji, Y.; Liu, Y.; Dan, Z.; Yang, P.; Zhu, Y.; Li, S. ORFH79 impairs mitochondrial function via interaction with a subunit of electron transport chain complex III in Honglian cytoplasmic male sterile rice. New Phytol. 2013, 198, 408-418. [CrossRef]

88. Liu, J.; Liang, L.; Jiang, Y.; Chen, J. Changes in Metabolisms of Antioxidant and Cell Wall in Three Pummelo Cultivars during Postharvest Storage. Biomolecules 2019, 9, 319. [CrossRef]

89. Ma, J.; Wei, H.; Song, M.; Pang, C.; Liu, J.; Wang, L.; Zhang, J.; Fan, S.; Yu, S. Transcriptome profiling analysis reveals that flavonoid and ascorbate-glutathione cycle are important during anther development in Upland cotton. PLOS ONE 2012, 7, e49244. [CrossRef]

(C) 2019 by the authors. Licensee MDPI, Basel, Switzerland. This article is an open access article distributed under the terms and conditions of the Creative Commons Attribution (CC BY) license (http://creativecommons.org/licenses/by/4.0/). 